function and a self-reported history of tuberculosis (airflow obstruction: adjusted odds ratio (aOR) 2.46 versus 2.51; spirometric restriction: aOR 2.11 versus 2.13).

We did indeed find, as Aggarwal and colleagues expected, that some participants had both obstructive and restrictive defects. In a sensitivity analysis, we excluded 482 participants who had both a forced expiratory volume in $1 \mathrm{~s} /$ forced vital capacity (FVC) ratio below the lower limit of normal (LLN) and FVC $<$ LLN, and showed that the associations were still present between tuberculosis and both obstruction and restriction. This demonstrates that there are independent associations between tuberculosis and these two outcomes.

Finally, Aggarwal and colleagues showed interest in knowing the proportion of participants with a history of tuberculosis and airflow obstruction who had symptoms. Of those with a history of tuberculosis and airflow obstruction, 54\% had wheeze and 33\% had modified Medical Research Council grade $\geq 2$ dyspnoea.

0 @ERSpublications

A history of tuberculosis is an important risk factor for obstructive disease and low lung function http://ow.ly/TXvgO

André F.S. Amaral ${ }^{1}$, Sonia Coton ${ }^{1}$, Bernet Kato ${ }^{1}$, Wan C. Tan ${ }^{2}$, Michael Studnicka ${ }^{3}$, Christer Janson ${ }^{4}$, Thorarinn Gislason $^{5}$, David Mannino ${ }^{6}$, Eric D. Bateman ${ }^{7}$, Sonia Buist ${ }^{8}$ and Peter G.J. Burney ${ }^{1}$

${ }^{1}$ Respiratory Epidemiology, Occupational Medicine and Public Health, National Heart and Lung Institute, Imperial College, London, UK. ${ }^{2}$ University of British Columbia Heart Lung Innovation Center, Vancouver, BC, Canada. ${ }^{3}$ Dept of Pulmonary Medicine, Paracelsus Medical University, Salzburg, Austria. ${ }^{4}$ Dept of Medical Sciences: Respiratory Medicine and Allergology, Uppsala University, Uppsala, Sweden. ${ }^{5}$ Faculty of Medicine, University of Iceland and Landspitali University Hospital, Reykjavik, Iceland. ${ }^{6}$ Division of Pulmonary Critical Care and Sleep Medicine, University of Kentucky, Lexington, KY, USA. ${ }^{7}$ Dept of Medicine, University of Cape Town, Cape Town, South Africa. ${ }^{8}$ Oregon Health and Sciences University, Portland, OR, USA.

Correspondence: André F.S. Amaral, Respiratory Epidemiology, Occupational Medicine and Public Health, National Heart and Lung Institute, Imperial College London, Emmanuel Kaye Building, 1B Manresa Road, London, SW3 6LR, UK. E-mail: a.amaral@imperial.ac.uk

Received: Aug 172015 | Accepted: Aug 182015

Conflict of interest: Disclosures can be found alongside the online version of this article at erj.ersjournals.com

\title{
References
}

1 Amaral AF, Coton S, Kato B, et al. Tuberculosis associates with both airflow obstruction and low lung function: BOLD results. Eur Respir J 2015; 46: 1104-1112.

\section{Number needed to treat: enigmatic results for exacerbations in COPD}

To the Editor:

SUISSA [1] provides the event-based number needed to treat (NNT) for a reduction in exacerbations with fluticasone/salmeterol compared with placebo for the TORCH (Towards a Revolution in COPD Health) trial by period of follow-up (0-1 years after treatment, 1-2 years after treatment and 2-3 years after treatment) [2]. These NNT data are misleading as they fail to recognise that in chronic obstructive pulmonary disease (COPD) trials, patients who exacerbate are more likely to withdraw from the trial than patients with no exacerbations. KEENE et al. [3] show that for the TRISTAN (TRial of Inhaled STeroids ANd long-acting $\beta_{2}$ agonists) trial, the exacerbation rate is more than three per year among placebo patients withdrawing prior to 1 year compared with an exacerbation rate of one per year for patients completing a year of placebo treatment. In the $\mathrm{TORCH}$ trial, $25 \%$ of the placebo group withdrew compared with $15 \%$ of the fluticasone/salmeterol arm during the first year of follow-up; therefore, patients entering the second year of the trial on placebo and on fluticasone/salmeterol were no longer directly comparable. This is not accounted for in the calculation of exacerbation rates or NNT during years 2 and 3 , as presented in table 2 of the study by SuISSA [1]. 
There are two other points that require further clarification. Firstly, table 2 incorrectly labels the treatment columns. These labels should be reversed; there was a higher rate of exacerbations per patient per year on placebo than on fluticasone/salmeterol. Secondly, in the introduction, the author quotes a previous paper [4] and states that the "NNT was illuminating in weighing up the benefit of inhaled corticosteroids in preventing COPD exacerbations against their risk of inducing pneumonia”. The methodology used to reach this conclusion based on NNT ignored the repeated nature of exacerbations [5].

In summary, as the author concludes, "it is important to ensure that the measures permit a comparison of like with like and are correctly calculated" [1]. The calculations for the TORCH trial presented by SuIsSA [1] fall short of these aims. For these reasons, we believe that the conclusions in SuIsSA [1] are not adequately supported by the data analyses presented.

$@$ ERSpublications

NNT calculations during years 2 and 3 are misleading as they don't account for differential withdrawal in year $1 \mathrm{http}: / /$ ow.ly/UeS61

Sally Lettis and Oliver Keene

Dept of Statistics, GSK, Uxbridge, UK.

Correspondence: Sally Lettis, Dept of Statistics, GSK, Stockley Park West, Building 10, 1-3 Iron Bridge Road, Uxbridge, UB11 1BT, UK. E-mail: sally.x.lettis@gsk.com

Received: Jan 102015 | Accepted: Aug 142015

Support statement: Funding information for this article has been deposited with FundRef.

Conflict of interest: Disclosures can be found alongside the online version of this article at erj.ersjournals.com

\section{References}

1 Suissa S. Number needed to treat: enigmatic results for exacerbations in COPD. Eur Respir J 2015; 45: 875-878.

2 Calverley PMA, Anderson JA, Celli B, et al. Salmeterol and fluticasone propionate and survival in chronic obstructive pulmonary disease. N Engl J Med 2007; 356: 775-789.

3 Keene ON, Calverley PMA, Jones PW, et al. Statistical analysis of exacerbation rates in COPD: TRISTAN and ISOLDE revisited. Eur Respir J 2008; 32: 17-24.

4 Suissa S. Number needed to treat in COPD: exacerbations versus pneumonias. Thorax 2013; 68: 540-543.

5 Keene ON, Anzeuto A, Ferguson GT, et al. Number needed to treat in COPD: exacerbations versus pneumonias. Thorax 2013; 68: 882.

Eur Respir J 2016; 47: 353-354 | DOI: 10.1183/13993003.01149-2015 | Copyright @eERS 2016

From the author:

S. Lettis and O. Keene raise questions about my paper on some misleading uses of the number needed to treat (NNT) for study outcomes such as chronic obstructive pulmonary disease (COPD) exacerbations. Certainly, when dealing with recurrent events such as exacerbations, it is statistically more informative to analyse all events with tools such as incidence rates, rate ratios and rate differences. However, some critical assumptions about the rates are essential to obtain valid estimates of these measures and, consequently, a valid estimate of the NNT.

Indeed, the underlying rates, estimated by the total number of exacerbations divided by the total time of follow-up, provide a mean number of exacerbations per year, such as two exacerbations per year over, for example, the 3-year follow-up of a trial. However, the statistics behind the estimation of this rate, be it the Poisson or negative binomial distribution, require that the rate be constant over the 3-year follow-up, which entails that the rate of two per year applies equally in the first, second and third year of follow-up. If not, rate differences may differ across the follow-up time and the resulting event-based NNT, computed by inverting the overall rate differences, will simply be incorrect.

My paper used the example of the 3-year Towards a Revolution in COPD Health (TORCH) trial to show why the reported NNT of 4 to prevent one exacerbation in 1 year is misleading [1]. A reader of the TORCH paper would logically understand this statement to mean that treatment of four patients for 1 year (any 1 year: the first, the last, etc.) leads to the prevention of one exacerbation. However, this is not the case. Indeed, it was quite arbitrary that the TORCH trial was a 3-year rather than, say, a 1-year trial. My paper shows that had TORCH been conducted as a 1-year trial, it would have reported a NNT of 0.5 to prevent one exacerbation in 1 year, not the NNT of 4 . Moreover, the paper shows that the NNT to 